\title{
A New Process of Color Images Recognition by Using ART-1 Neural Network
}

\author{
R.Thilepa (Corresponding author) \\ Department of EEE \\ Adhiyamaan Educational \& Research Institute, Hosur-Tamil nadu 635 109, India \\ Tel: 98-94-691-218 E-mail: thilepasudhakar@yahoo.co.in \\ M.Thanikachalam \\ Department of Civil Engineering \\ Velammal Engineering College, Chennai-600 066, India \\ Tel: 94-43-424-062_E-mail: thanikachalam84@yahoo.com
}

\begin{abstract}
In this paper ART1 neural network algorithm is used for recognition of color images which speeds up the process of Image processing and its recognition process. But, generally Adaptive Resonance Theory-1(ART-1) network is used only for processing binary digits, but in this paper it may also be used for image recognition. The given fact limits the loss of information as a result, two different colors in which inside one original image can be represented as a same value of binary color during recognition. These mistakes are overcome by our image recognition method here.This method is very attractive because of its low computational overhead, improved performance comparatively than other networks with less number of neurons. Experimental results are given finally which are encouraging in this paper.
\end{abstract}

Keywords: Image recognition, Neural Network, Adaptive Resonance Theory-1 network (ART-1)

\section{Introduction}

Image recognition is the process of obtaining an original perfect image from the degraded image. There are lots of reasons practically for degradation of an image and image recognition is one of the key fields in today's Digital image processing due to its wide area of applications. The commonly occurring degradation of pictures is due to blurring, motion and noise. The ART1 network we are using here is used to recognize lot of colors but it causes lot of complexity and processing time required is more. Therefore in order to reduce the processing time, we have converted a sequential structure of the ART1 network to the structure that may be implemented with N number of independent processors (Carpenter, G.A. and S. Grosberg, 1987)(Faulset, L. 1994).

The initial step in conversion of ART1 neural network is parallel computing using network decomposition. Generally an ART1 network contains three layers accordingly as (i) input layer,(ii) interface layer and (iii) recognition layer. In this network number of input vectors, number of clusters and number of parallel processors are assumed. The only limitation provided here is the ratio of number of clusters to number of tasks should be an integer. Then for this parallel processing system we provide a sequential system. The processor provided here will act as source for data and also serve for synchronization process. Hence data distribution is provided between all members of the network in the system.

The sequential learning algorithm is used generally in ART1 network. But here in our paper we have provided a modified different algorithm for parallel processing. The main methods involved in this algorithm are (i) Training Algorithm computing method and (ii) Learning Algorithm computing methods which are explained below. Thereby Image recognition represented by many publications where ART1 can be used for complex images recognition by composing reference images without learning all combinations of the reference images.

\section{Why Use ART-1 NN?}

- $\quad$ ART1 neural network has a parallel structure and generalizes almost better than any other neural network.

- In general the length of input vector is less than the number of clusters in recognition layer.

- These qualities make ART1 Network more feasible for real time applications such as image recognition. 
- This ART1 network involves only two main steps that is learning the network and image recognition.

3. Steps Involved In Working with ART-1 NN

- The given network is decomposed as shown in fig (a)

- The process is divided into two main steps that are execution of training algorithm and clustering algorithm.

- The fig (a) consists of $\mathrm{R}$ as reset unit along with $\mathrm{b}_{\mathrm{ij}}$ as bottom-up weights and $\mathrm{t}_{\mathrm{ji}}$ are top-down weights.

- The network consists of n-size of input vector with m-number of clusters in recognition unit along with $\mathrm{N}$-number of tasks.

4. Methodology

- According to the process the ratio of $\mathrm{m} / \mathrm{N}$ should be an integer number.

- Accordingly learning the network (execution of training algorithm) is done first.

- Later image recognition (clustering algorithm) is done.

The Fig (2a) figure shows the A-Training Algorithm and B-recognition Algorithm

Let us consider the notations

$\mathrm{N}=$ number of components in input vector

$\mathrm{m}=$ Maximum number of cluster that can be formed

$b_{i j}=$ Bottom up weights (from interface to recognition layer)

$\mathrm{t}_{\mathrm{ji}}=$ Top down weights (from recognition layer to interface layer)

$\mathrm{S}=$ Binary input vector

$\mathrm{X}=$ Activation vector for interface layer

$\mathrm{N}=$ Number of processors

Training Algorithm Computing Method:

- First we have to solve the training algorithm, in this we have to check the number of process, if the process isroot a training set is loaded from the files.

- The training set of data is loaded to all the members of the system.

- In the other case if the process is not zero then, all F2 units are set to zero and F1 (a) units to input vector $\mathrm{S}$.

- Now compute $\mathrm{S}$, then an input signal is sent from input to interface layer.

- For each node compute

$$
y_{j}=\sum_{i} b_{i j} x_{i}, \quad \text { Here } x_{i}=s_{i} t_{i j}
$$

In this method thereby computation and update of matrices takes less number of iterations compared to other methods.

\section{Learning Algorithm Computing Method:}

- In this algorithm again the rank is checked if it is equal to zero then the image is loaded from the file.

- Similarly the image is broadcasted between all the members of the system.

- The response of cluster units are computed in the recognition layer as $\mathrm{m} / \mathrm{N}$ single processor as follows,

For $\mathrm{j}=\mathrm{N}_{1}$ to $\mathrm{N}_{2}$ follow external loop \& go to step 1

For $\mathrm{i}=1$ to $\mathrm{n}$ follow internal loop\& go to step 1

Begin

$$
y[j]:=y[j]+b[j, i] * x[i]
$$

end;

Here

$\mathrm{N} 1=\mathrm{m} / \mathrm{N} * \operatorname{rank}$ and $\mathrm{N} 2=\mathrm{m} / \mathrm{N} *(\operatorname{rank}+1)-1$

Where $\mathrm{N}_{1}$ and $\mathrm{N}_{2}$ are number of iterations.

- In this step the reduction process is done and then each processor returns values of cluster units $y_{j}$ to the root that gathers required information to the single vector.

- The root analyses a vector $y_{\mathrm{j}}$ and searches the number of excited neurons and gives out the practical results. 
- The practical part is divided into two parts such that ability of testing the ART1 network in recognition of color images in the first stage and in the next stage speeding up evaluation for parallel implementation of training and recognition of images.

The different colors such as shown above in ART1 network in this paper is analyzed using Borland Delphi 6.0 compiler (Kerman, F. and C. Miitchell, 2004)(Rachele). Here the program allows up to 200 input vectors ranging from 0.1 to 1 with maximum number equal to 200 .

For our implementation the input vector is chosen such that in 64 pixels and can be able to process $8 * 8$ images. The below Fig (d) shows the simulated output of an image that is taken for analysis. The table Fig (e) given below also provides the details of recognition ability of the network. For simplification purposes three pixels per pixel color coding here. For obtaining accuracy we have taken Latin Alphabets A...Z which includes four different colors, then totally 104 images can be processed in BMP format.

The below fig (e) shows the simulated output of the program. Here during the training stage the input is fed to the network as input. In the recognition stage the initial number of files are shuffled in random order and then sent to input again. The input of the network is given as follows:

(i) Input vector size $=8 * 8$

(ii) Vigilance parameter

(iii) Maximum number of clusters $=104$

(iv) Source of bit image-external bitmap files

For our analysis we have taken two microscopic images of staphylococcus aureus and streptococcus pyogenes and is shown below

In our analysis three tests are done such that in lower vigilance parametersalso it is able to recognize the color of pattern.In this test Red,Blue,Green A characters are recognised correctly and yellow characters (character number 3) clustered as (character number 4).In this test the binary value of each character is different.then second test is done here all the characters are analysed with moderate vigilance parameters.Finally third test is done where interpolation of network is done hence pattern 8 (dark grey) interpolated as pattern 5(black).

The statistical sensitivity is calculated to obtain the recognition ability of the network(Liu, J. and Y.H. Yang, 1994)( Chatfield, C., 2005). Hence

\author{
Sensitivity $=$ Number of true positives \\ Number of true positives + Number of false negatives
}

Finally the results obtained gives that the network is able to recognise different images or objects ranging in the vigilance parameter from 0.7-0.8.If the vigilance value is reduced inturn sensitivity is reduced.Next parallel implementation of ART1 network is done in disributed memory parallel systems.For this purpose we have taken MPICH system (version 1.2.5 for NT) in construction of parallel processing clusters. The cluster includes 8 systems in sequential order.The program implemented a parallel realization of ART1 training and recognition algorithm created by Visual $\mathrm{C}++2005$ compiler,console application,the model of the parallel processing system-SPMD(Carpenter, G.A. and S. Grosberg, 1987).This program was made to count the microorganisms with high resolution images(Madigan, M.T., J. Martinko and J. Parker, 2004).Based on these highly resoluted images a training set for neural network is formed.

Both bacterias here we have taken for analysis are of same form but they differ only in colors according to different Gram's method(Madigan, M.T., J. Martinko and J. Parker, 2004).We have taken 12 patterns in each category of bacteria for analysis(for I pattern 1-12 \& II pattern 13-24).Each pattern we have taken has a resolution of $36^{*} 36$ pixels and the length of vector equal to 3888 bits. This program was distributed to all the sequential systems and the evaluation of time for training and recognition algorithms for the network is done. The number of processors inside the cluster are :

(a) Single sequential system -1

(b) Distributed parallel processing system-2,3,4,6 and 8 .

Here the numbers 5 and 7 are skipped due to the limitation of parallel algorithms. The speed up of parallel processing system is obtained by

Speed-Up $=$ Time taken for task execution for sequential program

Time taken for task execution for parallel program

The below Fig $(\mathrm{g})$ shows the relationship between number of processors and speed up times. 
The evaluation of execution time is done according to standard MPI function MPI_Wtime(),and the measurement of time is provided after broadcasting of training sets and recognizable images. Therby parallel implementation of ART1 network is analysed by taking two different bacteriasin parallel implementation process.

\section{Conclusion}

Generally ART1 network is used for binary input processes.It plays a least role in color image processing since, the images has to be converted into binary datas when this process is done there is possibility of information loss.But by using our parallel process the information loss is reduced in an enormous way and also the processing time is reduced.The sensitivity of the network is more in this case comparitively and the vigilance risks are reduced by this method.Thus in our paper we have elaborated a new color image recognition method.Further development of this suggested method applications lies in speeding up of the color image recognition method.

\section{References}

Carpenter, G.A. and S. Grasberg. (1987). Massivelyparallel architecture for self-organizing Neuralpattern recognition, Machine// computer vision.Graphics and Image Processing, 37: 45115.

Faulset, L. (1994). Fundamentals of NeuralNetworks. Architectures, Algorithm and Applications. New Jersey, Prentice Hall Int.,pp: 461.

Kerman, F. and C. Miitchell. (2004). Programmingand Problem Solving With Delphi. UK INTERNATIONAL ED, Pearson Higher EducationDate Published: ISBN: 0321204417. Rachele, Warren Learn Object Pascal with Delphi.Wordware Publishing inc. ISBN: 1556227191.

Madigan, M.T., J. Martinko and J. Parker. (2004). Brock Biology of Microorganisms, 10th Edition,Lippincott Williams and Wilkins.ISBN 0-13-066271-2.

Liu, J. and Y.H. Yang. (1994). Multiresolution color image segmentation. IEEE Trans. Pattern Anal. Mach. Intell. 16 (7): 689-700.

Chatfield, C. (2005). Statistics for Technology: A Course in Applied Statistics. Third Edition CRC press. ISBN: 0412253402 .

Gonzalez, R.C. and R.E. Woods. (1992). Digital Image Processing. Reading, Massachusetts: Addison-Wesley, pp: 716.

Kwang-Baek Kim, Kwee-Bo Sim, Sang-Ho Ahn. (2006). Recognition of Concrete Surface Cracks Using thART1-Based RBF Network // Advances in Neural Networks - ISNN 2006, Volume 3972/2006, p.669-675.

Task 0

Task 1

Task N-1

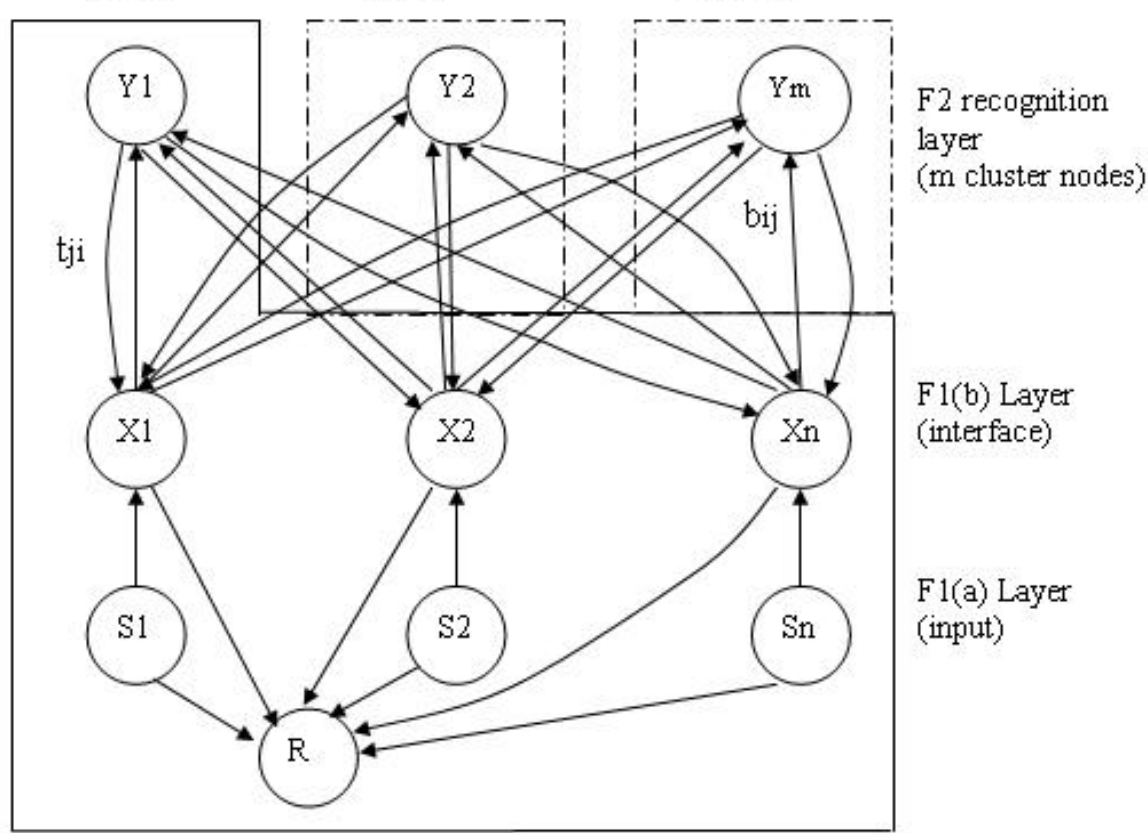

Figure (a). ART1 Neural Network with Decomposition 

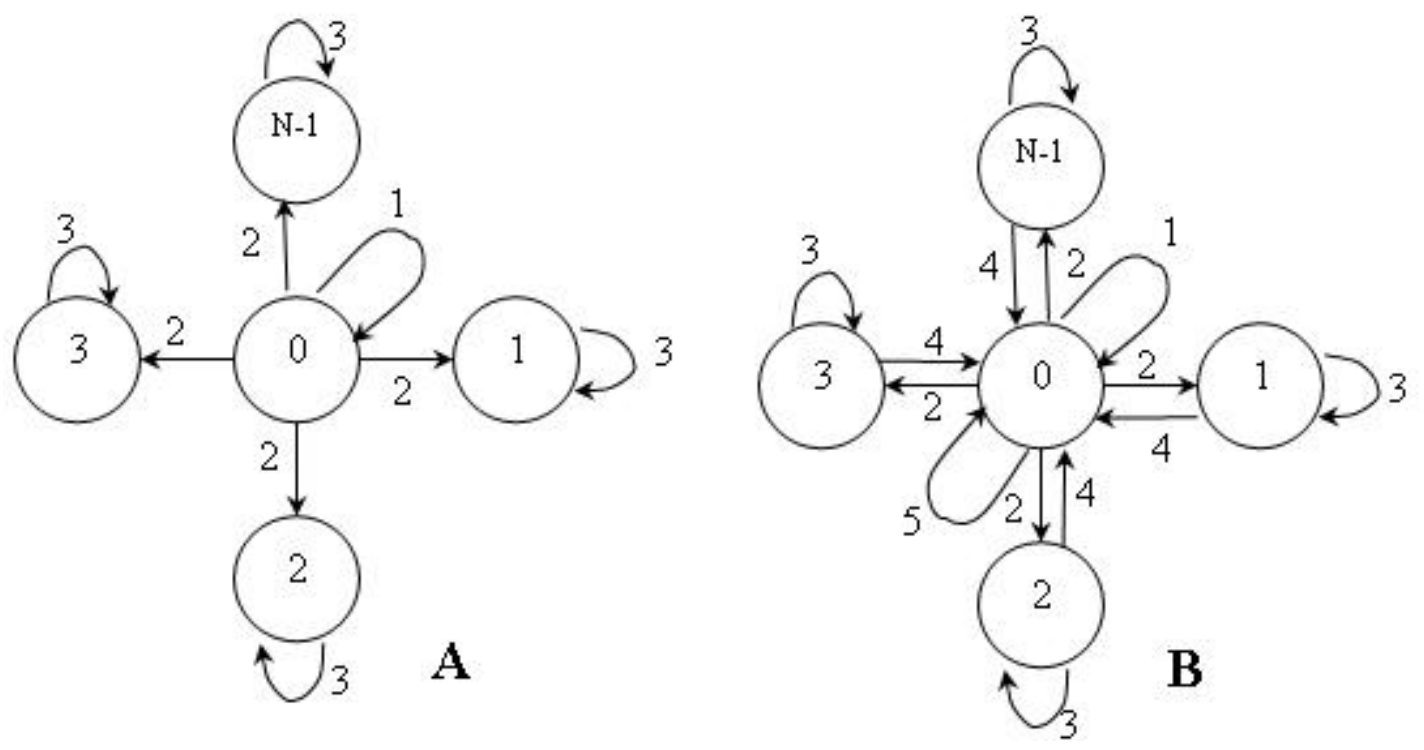

Figure (2a). Training Algorithm \& Learning Algorithm

\section{True colors (24 bits)}

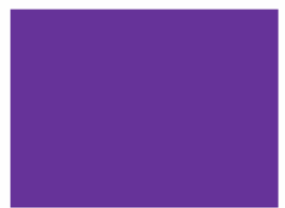

$\mathrm{R}=96$

$G=50$

$B=150$

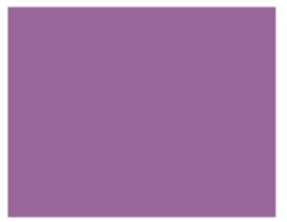

$\mathrm{R}=150$

$G=100$

$\mathrm{R}=220$

$\mathrm{B}=150$

$G=190$

$B=90$

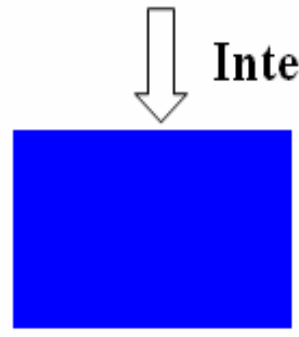

Interpolated colors

$\mathrm{R}=0$

$G=0$

$B=1$

Blue

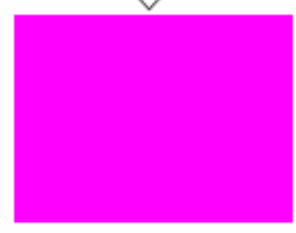

$R=1$
$G=0$
$B=1$
Violet

$\mathrm{R}=1$

$G=1$

$\mathrm{B}=0$

Yellow

Figure (b) Interpolated colors 


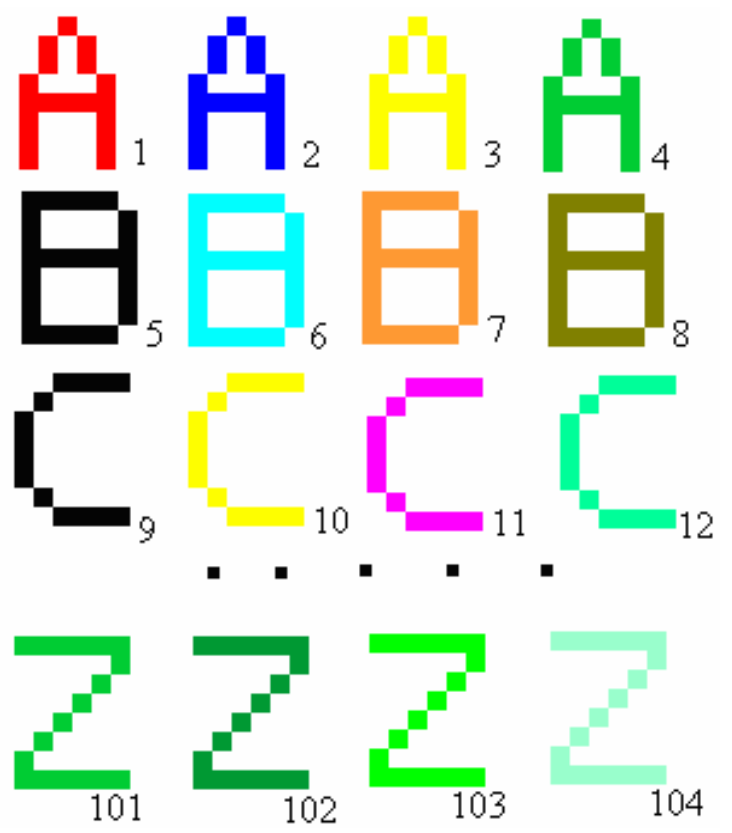

Figure (c) Training and Recognition set

Table 1. Recognition Ability Table

\begin{tabular}{|c|c|c|c|c|c|}
\hline $\begin{array}{c}\text { Test } \\
\text { Number }\end{array}$ & $\begin{array}{c}\text { Vigilance } \\
\text { parameter }\end{array}$ & Learning sequence & Input sequence & $\begin{array}{c}\text { The network } \\
\text { outputs }\end{array}$ & Note \\
\hline 1 & 0.4 & $1,2,3,4$ & $1,4,3,2$ & $1,3,3,2$ & $\begin{array}{c}\text { Small vigilance parameter } \\
\text { causing clusters into 3 } \\
\text { clusters }\end{array}$ \\
\hline 2 & 0.75 & $1,2,3,4$ & $1,4,3,2$ & $1,4,3,2$ & $\begin{array}{c}\text { All patterns recognized } \\
\text { correctly }\end{array}$ \\
\hline 3 & 0.75 & $5,6,7,8$ & $8,6,5,7$ & $5,6,5,7$ & $\begin{array}{c}\text { Pattern 8 (dark grey) is } \\
\text { recognized as pattern 5 } \\
\text { (black) }\end{array}$ \\
\hline 4 & 0.75 & $101,102,103,104$ & $104,101,103,102$ & $102,101,101$ & $\begin{array}{c}\text { All patterns clustered into } \\
\text { two clusters }\end{array}$ \\
\hline
\end{tabular}

Figure (d) Testing the Recognition ability of the network 


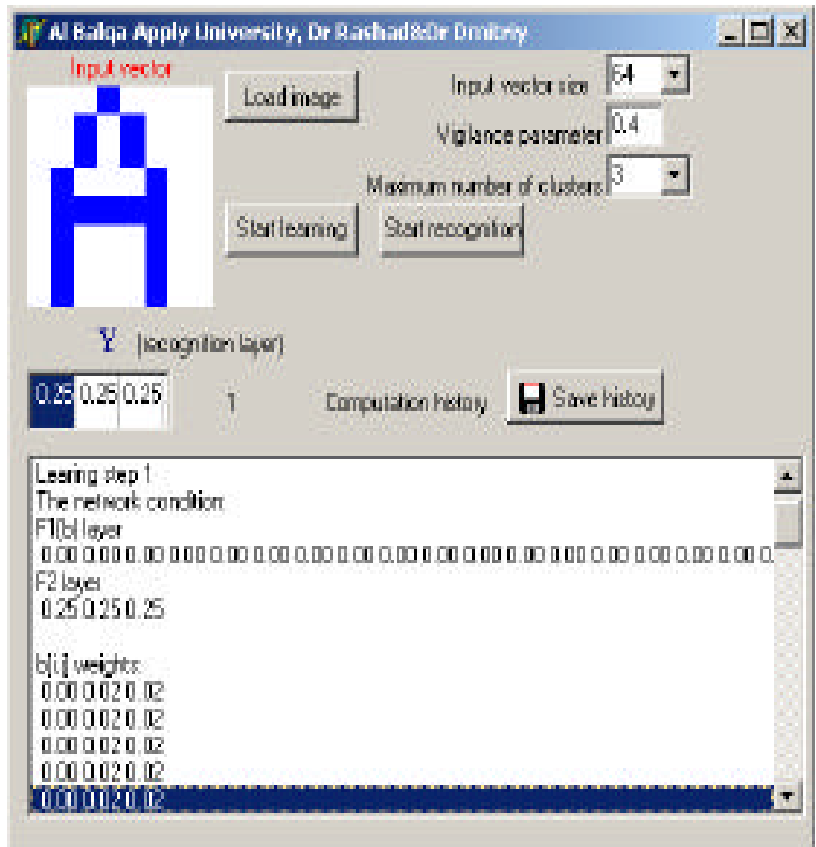

Figure (e) GUI of Designed Program

Description of

Object
No. of training

sets

\section{Images}

Staphylococcus aeurus

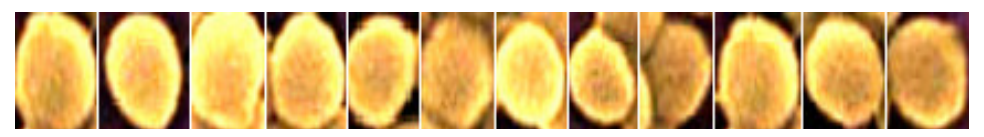

Streptococcus pyogenes 13-24

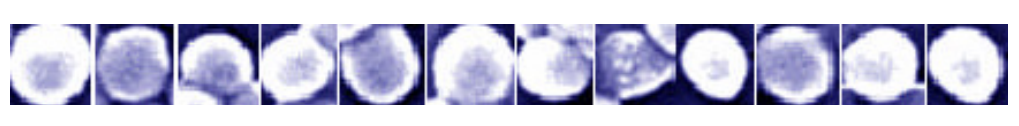

Figure (f) Training set for recognition of microscopic images

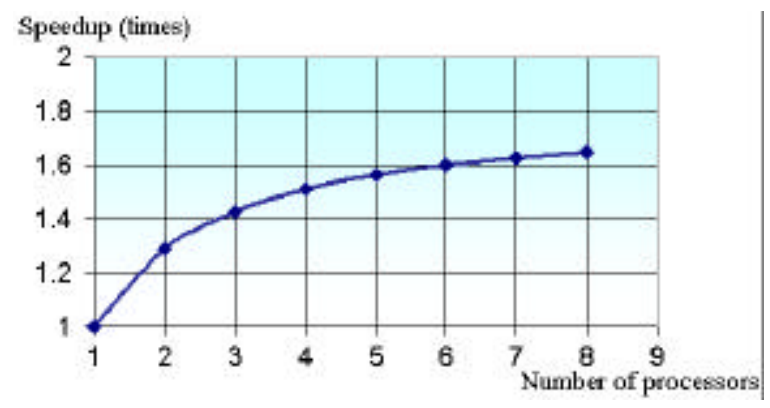

Figure (g) Relation between number of processors and speed-up times 